\title{
Spatial Resolution of Infrared Imaging Systems
}

\author{
Alla V. Shymanska* \\ School of Computer and Mathematical Sciences, Auckland University of Technology, Private Bag 92006, \\ Auckland 1142, New Zealand. \\ * Corresponding author. Email: alla.shymanska@aut.ac.nz \\ Manuscript submitted June 12, 2016; accepted August 2, 2016. \\ doi: 10.17706/ijapm.2016.6.4.207-217
}

\begin{abstract}
The image quality and the resolving power of an optical device can be improved by improving its modulation-transfer-function (MTF). It is especially important for the devices which are designed for conditions of low illumination, such as a night vision. The case of infrared image converters and intensifiers with an inverting electron-optical system (EOS) and a micro-channel plate (MCP) as an amplifier is taken for consideration in this work. The electron amplification process in the channel is simulated by Monte Carlo methods. As a result, the energy and angular distributions are determined and used as initial conditions for calculation of the current density distribution in the channel image on the screen. The effect of the electrostatic field penetration into the channel on the current-density distribution and the MTF of the system is under investigation in this work. The effect of the length of the contact conducting layer on the field penetration and MTF is evaluated. It is also shown how the emission from the conducting layer affects the resolution. The optimal parameters of EOS which provide a flat image surface to coincide with the channel plate are defined. Position of the best focus in the electron beam, what provides the highest resolution, is determined. Finally, the total MTF of the imaging device is calculated.
\end{abstract}

Key words: Image device, modulation-transfer-function, microchannel electron amplifier, Monte Carlo simulations.

\section{Introduction}

Distinction of small details of an object, and therefore, the range of vision of any image detector, are limited by optical properties of the detector, which are usually characterized by a modulation-transfer-function (MTF) [1]. The MTF describes the resolution over the image area of an image device, and gives the dependence of the output contrast on the spatial frequency. The image quality, and the resolving power of the device can be improved by improving its MTF. It is especially important for the devices which are designed for conditions of low illumination, such as a night vision. The case of infrared image converters and intensifiers with an inverting electron-optical system (EOS) and a micro-channel plate (MCP) as an amplifier is taken for consideration in this work [2], [3].

The process of image conversion and intensification in such devices is evaluated as follows: (a) electromagnetic radiation from a certain part of the spectrum is absorbed by a photocathode; (b) an optical image is converted into an electron image by means of the photoemission; (c) the photoelectrons of this electron image are accelerated and focused by an electron optical system, and intensified by the MCP; (d) the intensified electron image is converted into a visible image by a luminescent process on a screen.

The MTF of the overall system, at a given spatial frequency, is the product of the MTFs of the elements. 
Consequently, to evaluate the total MTF of the imaging system with the MCP, the MTFs of the electron-optical system and MCP-screen system should be determined.

MCP is a thin plate made up of a large number of single channels, each of which acts as an electron multiplier [4]. A single channel multiplier consists of cylindrical dynode having a small diameter. For each primary electron entering a channel, a large pulse of electrons will exit as a result of multiple collisions of both primary and secondary electrons with the inner secondary-emission layer as they traverse the length of the channel (Fig. 1). It is a compact, efficient amplifier of two-dimensional electron-images and provides several advantages such as very high gain, good resolution and small size.

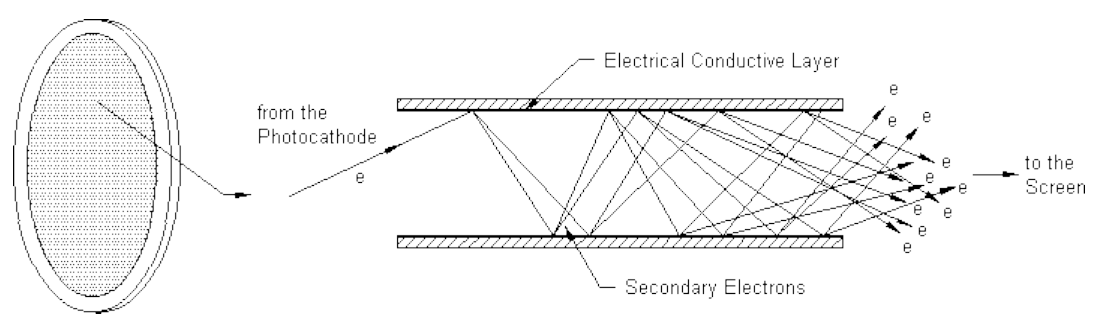

Fig. 1. MCP and electron multiplication in the channel.

The MCP is placed behind the anode diaphragm close to the screen. To accelerate the secondary electrons toward the exit of the channel, the voltage is applied by depositing a contact conducting layer at its ends.The electrostatic field of the MCP-screen gap penetrates into the channel and creates an electrostatic lenswhich affects the electron trajectories, and consequently, the current-density distribution on the screen at the MCP exit, and therefore, the image quality on the screen [5], [6]. The potential distribution in the field of lens depends on the field intensity in the MCP-screen gap and in the channel, the channel diameter size, and the sputtering depth of the contact conducting layer at the channel output. The current density distribution in the image of an individual channel and its MTF are determined from the arrival coordinates of the electrons on the screen which depend on energy and angular distributions of the electrons that come out of the channel.

Calculation of the channel amplifier's MTF requires a simulation of complicated stochastic processes of the secondary electron emission inside the channel, where energy, angular and the current-density distributions are determined. It also requires to calculate anon uniform field at the exit of the channel and in the MCP-screen gap, and trajectories of the electrons in such field.

For the best image quality on the screen the EOS should provide a flat image surface to match the micro channel plate. Also, the position of the surface of the best focus, where the spatial resolution is the highest, should coincide with the MCP position. Such analysis includes numerical calculation of the field distribution inside the EOS and trajectories of electrons emitted from the photocathode.

In this work the electron amplification process in the channel is simulated by Monte Carlo methods [7], [8] where electron trajectories in uniform electrostatic field inside the channel and in non uniform field at its end are calculated from the relevant equations of motion. As a result, the energy and angular distributions are determined and used as initial conditions for calculation of the electron trajectories in the MCP-screen gap. The resulting distribution of the arrival coordinates of the electrons on the screen determine the current density distribution in the channel image. The effect of the electrostatic field penetration into the channel on the current-density distribution and the MTF of the system with MCP is under investigation in this work. The effect of the length of the contact conducting layer on the field penetration and MTF is evaluated. It is also shown how the emission from the conducting layer affects the resolution.

Trajectories of the photoelectrons in the non uniform electrostatic field of the EOS are calculated to find coordinates of the minimal cross section of the electron beams from the cathode [3]. Thus, the curvature of 
the image surface is determined as a total combination of minimal cross sections of the electron beams, emitted from different points of the photocathode. The optimal parameters of EOS which provide a flat image surface to coincide with the channel plate are defined. Position of the best focus in the electron beam, what provides the highest resolution, is determined. Finally, the total MTF of the imaging device is calculated.

\section{Computational Methods}

\subsection{Motion of Electrons in the Potential Field}

Calculation of the electrostatic field in the device with rotational symmetry (Fig. 2 and Fig. 3) is a matter of finding a solution to the Laplace's partial differential equation expressed in cylindrical coordinates [3]:

$$
\frac{\partial^{2} U}{\partial z^{2}}+\frac{1}{r} \frac{\partial U}{\partial r}+\frac{\partial^{2} U}{\partial r^{2}}=0
$$

where $U(r, z)$ is the potential at any point $(z, r), z$ is the coordinate along the axis of symmetry and $r$ is the off-axis radius.

It is the classical mixed problem for the equation of Laplace in some region with Dirichlet and Neumann boundary conditions. The finite difference method is used to obtain the field distribution.

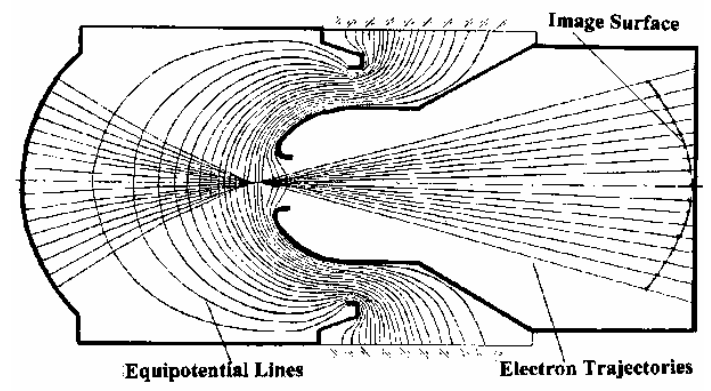

Fig. 2. Equipotential lines and central meridional trajectories of electron beams.

Fig. 2 shows the computational results of the potential distribution (given by the equipotential lines) in the cross-section of the EOS. Fig. 3 shows the computational results of the electrostatic lens at the exit of a single channel.

In the cylindrical coordinate system, equations of motion of electrons can be written as:

$$
\begin{aligned}
& \frac{d^{2} z}{d t^{2}}=\frac{e}{m} E_{z} \\
& \frac{d^{2} r}{d t^{2}}=\frac{e}{m} E_{r}+\frac{r_{0}^{2} V_{\varphi 0}^{2}}{r^{3}}, \\
& \frac{d \varphi}{d t}=\frac{r_{0}}{r^{2}} V_{\varphi 0}
\end{aligned}
$$

where $t$ is time, $E_{r}=\frac{\partial U}{\partial r}$ and $E_{z}=\frac{\partial U}{\partial z}$ are the radial and axial components of the strength of the electrostatic field respectively, $r_{0}$ is the initial electron coordinate, $V_{\varphi 0}$ is the initial azimuthal 
component of the electron velocity, $e$ and $m$ are electron charge and mass respectively.

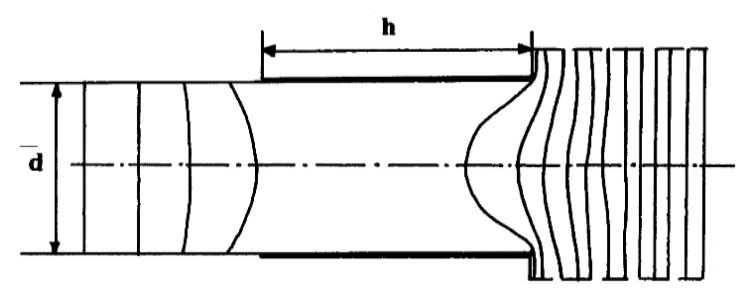

Fig. 3. Electrostatic field at the exit of the channel.

The system of equations (2) is solved by the Runge-Kutta method for the electron motion inside the EOS of the device, and in the area of the inhomogeneous electrostatic field of the channel multiplier. The strengths of the electrostatic field at the exit of a single channel and inside the EOS are calculated using different interpolating polynomials [3]. Fig. 2 shows central meridional trajectories of the electron beams emitted from the photocathode.

\subsection{Simulation of Stochastic Processes}

The process of Monte Carlo simulations uses a random number generating procedure [7], [8] to sample the various distributions such as: the distribution of the actual yield of secondary electrons after each collision, the emission energy, and the direction of each secondary electron.

The actual number of secondaries generated by the particular collision is a random sample taken from the Poisson distribution $P(v)=\frac{\sigma^{v} e^{-\sigma}}{v !}$, where $v$ is the number of secondary electrons produced, $\sigma$ is the Secondary Emission Yield (SEY). The variation of the SEY is defined by a secondary emission function [7]:

$$
\sigma=\sigma_{m}\left[\frac{V}{V_{m}} \sqrt{\cos \theta_{0}}\right]^{\beta} e^{\alpha\left(1-\cos \theta_{0}\right)+\beta\left(1-\frac{V}{V_{m}} \sqrt{\cos \theta_{0}}\right)},
$$

where $V$ is the collision energy in $\mathrm{eV}, \theta_{0}$ is the collision angle (angle between the direction of the primary electron and the normal to the surface), $\sigma_{m}$ is the maximum SEY for the normal incidence $\theta_{0}=0$ ) which is achieved for $V=V_{m}$ (both $V_{m}$ and $\sigma_{m}$ are functions of $\theta_{0}$ ); $\alpha$ and $\beta$ are constants of the channel multiplier surface, and are chosen to fit experimental secondary emission curves at normal incidence.

The energy distribution is described by the formula [9]:

$$
p(\varepsilon)=2.1 \bar{\varepsilon}^{-3 / 2} \sqrt{\varepsilon} \exp (-1.5 \varepsilon / \bar{\varepsilon}) \text {, where } \bar{\varepsilon} \text { is the mean energy. }
$$

Each secondary electron is assigned two emission angles chosen from the Lambert's law:

$p_{1}(\theta)=\sin 2 \theta$ and $p_{2}(\varphi)=1 / 2 \pi$, where $\theta$ is the angle between the normal to the surface and emission direction, and $\varphi$ is the azimuthal angle.

The trajectory of each electron is calculated in three dimensions from the equations (2), and the position, energy, and angle of the subsequent collisions are determined. The result of each collision is calculated as before and the process is repeated for each secondary electron generated.

\subsection{Modulation Transfer Function of the Image System}


The MTF in the image plane for the point on the axis of the symmetry of EOS is calculated using the formula [10]:

$$
T=\frac{\int_{0}^{\infty} A(r) J_{0}(2 \pi v r) r d r}{\int_{0}^{\infty} A(r) r d r}
$$

where $J_{0}$ is the Bessel's function, $v$ is a spatial frequency, and $A(r)$ is an electron density distribution in the cross section of the axial electron beam on the image surface.

To find the electron density function $A(r)$ let assume that $d N(\varepsilon, \theta, \varphi)$ electrons are emitted from the axial point of the photocathode in a time unit with initial energies in the interval $[\varepsilon, \varepsilon+d \varepsilon]$, initial angles $[\theta, \theta+d \theta]$ and $[\varphi, \varphi+d \varphi]$, where $\theta$ and $\varphi$ are meridional and azimuthal angles respectively.

The number of electrons $d N(\varepsilon, \theta, \varphi)$ depends on the total number of the electrons $N_{0}$ emitted from one point of the photocathode and the probability of the emission $P(\varepsilon, \theta, \varphi)$. Therefore,

$$
d N(\varepsilon, \theta, \phi)=N_{0} d P(\varepsilon, \theta, \phi)=N_{0} p(\varepsilon, \theta, \phi) d \varepsilon d \Omega
$$

where $p(\varepsilon, \theta, \varphi)$ is the probability density, and $d \Omega=\sin \theta d \theta$.

Since $\varepsilon, \theta$ and $\varphi$ are independent random variables then $p(\varepsilon, \theta, \varphi)=p(\varepsilon) p(\theta) p(\varphi)$, where $p(\varepsilon), p(\theta)$, and $p(\varphi)$ are probability density functions of the electron's energy, meridional and azimuthal angles respectively. Assuming that $p(\varphi)=2 \pi$ is the uniform distribution, the expression for the number of the emitted electrons $d N(\varepsilon, \theta, \varphi)$ can be written as:

$$
d N(\varepsilon, \theta, \varphi)=N_{0} p(\varepsilon) d \varepsilon p(\theta) \sin \theta d \theta \int_{0}^{2 \pi} p(\varphi) d \varphi=N_{0} p(\varepsilon) p(\theta) \sin \theta d \theta d \varepsilon
$$

The photoelectrons with the initial energy $\varepsilon$ would have a distance $r$ from the axis of the symmetry at the image surface if their initial angle $\theta$ satisfies a condition $r-f(\varepsilon, \theta)=0$. Using the $\delta$-function the expression for the electron density at the distance $r$ from the axis of the symmetry can be written as:

$$
d n(r)=\frac{d N(\varepsilon, r)}{2 \pi r}=\frac{N_{0} p(\varepsilon)}{2 \pi r} \int_{0}^{\pi / 2} p(\theta) \sin \theta \delta[r-f(\varepsilon, \theta)] d \theta d \varepsilon
$$

Integrating (4) with respect to energy $\varepsilon$ the electron density function $A(r)$ can be expressed as

$$
A(r)=\int_{0}^{\varepsilon_{0}} \frac{N_{0} p(\varepsilon)}{2 \pi r} \int_{0}^{\pi / 2} p(\theta) \sin \theta \delta[r-f(\varepsilon, \theta)] d \theta d \varepsilon
$$

Substituting (5) to (3) the expression for the modulation transfer functions can be written as:

$$
T(v)=\frac{\int_{0}^{\infty} \int_{0}^{\varepsilon_{0}} p(\varepsilon)\left\{\int_{0}^{\pi / 2} p(\theta) \sin \theta \delta[r-f(\theta, \varepsilon)] d \theta\right\} d \varepsilon J_{0}(2 \pi v r) d r}{\int_{0}^{\infty} \int_{0}^{\varepsilon_{0}} p(\varepsilon)\left\{\int_{0}^{\pi / 2} p(\theta) \sin \theta \delta[r-f(\theta, \varepsilon)] d \theta\right\} d \varepsilon d r}
$$


Using the property of the $\delta$-function that $\int_{0}^{\infty} \varphi(x) \delta\left(x-x_{0}\right) d x=\varphi\left(x_{0}\right)$ the final formula for the modulation transfer functions can be obtained:

$$
T(v)=\frac{\int_{0}^{\varepsilon_{0}} p(\varepsilon) \int_{0}^{\pi / 2} p(\theta) \sin \theta J_{0}[2 \pi v f(\theta, \varepsilon)] d \theta d \varepsilon}{\int_{0}^{\varepsilon_{0}} p(\varepsilon) \int_{0}^{\pi / 2} p(\theta) \sin \theta d \theta d \varepsilon},
$$

where $p(\varepsilon), p(\theta)$ are energy and angular distributions of the electrons respectively (here the angular distribution is described by Lambert's law, $p(\theta)=\cos \theta$, and the electron energy is chosen from the parabolic function $p(\varepsilon)=\frac{6 \varepsilon}{\varepsilon_{0}}\left(1-\frac{\varepsilon}{\varepsilon_{0}}\right) ; \varepsilon_{0}$ is the maximal possible initial energy of the photoelectron; $r=f(\varepsilon, \theta)$ is the distribution of the electron radial coordinates at the image surface which can be written as:

$r(z)=2 \sqrt{\frac{\varepsilon}{E_{0}}} \sin \theta u_{1}(z)-\frac{2 \varepsilon}{E_{0}} \sin \theta \cos \theta u_{2}(z)$, where $E_{0}$ is the strength of the electrostatic field near the photocathode; $u_{1}(z)$ and $u_{2}(z)$ are two particular solutions of the equation of the motion of paraxial electrons. $u_{1}(z)$ and $u_{2}(z)$ are calculated as described above.

Formula (3) is used in computations of the MTF of the MCP-screen system as well. The electron density distribution $A(r)$ in the image of an individual channel is determined from the arrival coordinates of the electrons on the screen.

Taking into account the discreet nature of the determination of $A(r)$, and using the relationship $\int x^{n} J_{n-1}(x) d x=x^{n} J_{n}(x)$, the calculating formula for MTF of the channel multiplier can be written as:

$$
T=\frac{\sum_{i=1}^{n} A_{i}\left(r_{i}\right)\left[r_{i+1} J_{1}\left(2 \pi v r_{i+1}\right)-r_{i} J_{1}\left(2 \pi v r_{i}\right)\right]}{\pi v \sum_{i=1}^{n} A_{i}\left(r_{i}\right)\left(r_{i+1}^{2}-r_{i}^{2}\right)},
$$

\section{Computational Results}

\subsection{Spatial Resolution of the Channel Amplifier}

The use of MCP as an amplifier in image devices has many advantages, but the discrete structure of the MCP and defocusing of the electron beam in the space between the MCP and the screen, restricts the spatial frequency what can be transferred by the device. A meaningful MTF can be obtained up to the array frequency [1]. The loss in contrast due to the divergence of the electrons in the MCP-screen gap can be reduced by carefully designing the electron lens at the output of each channel. The lens is formed by the penetration of the gap field into a channel [5], [6]. The limit is set by the smallest gap that will reliably hold of the voltage necessary to excite the phosphor of the screen.

The current density distribution in the image of the individual channel on the screen, is determined by 
the energy and angular distributions of the electrons, emerging from the channel, and depends on how the electrostatic lens at the output of the channel focuses the electron beam.

The multiplication process of the electron flux in the channel was modelled as it is described in section 2.2, and for electrons, leaving the channel, the energy, angle and the arrival coordinates were determined. Calculated energy and angular distributions for the electrons exiting the channel are shown in Fig. 4 and Fig. 5 respectively, and are well agree with data in [7], [11]. Computational results were obtained for the following channel parameters: diameter $d=10 \mu \mathrm{m}$, voltage on the channel $V=800 \mathrm{~V}$, strength of the electrostatic field in the MCP-screen gap $E=5 \mathrm{kV} / \mathrm{mm}$, and the length of the contact conducting layer at the end of the channel $h=1.5 d$.

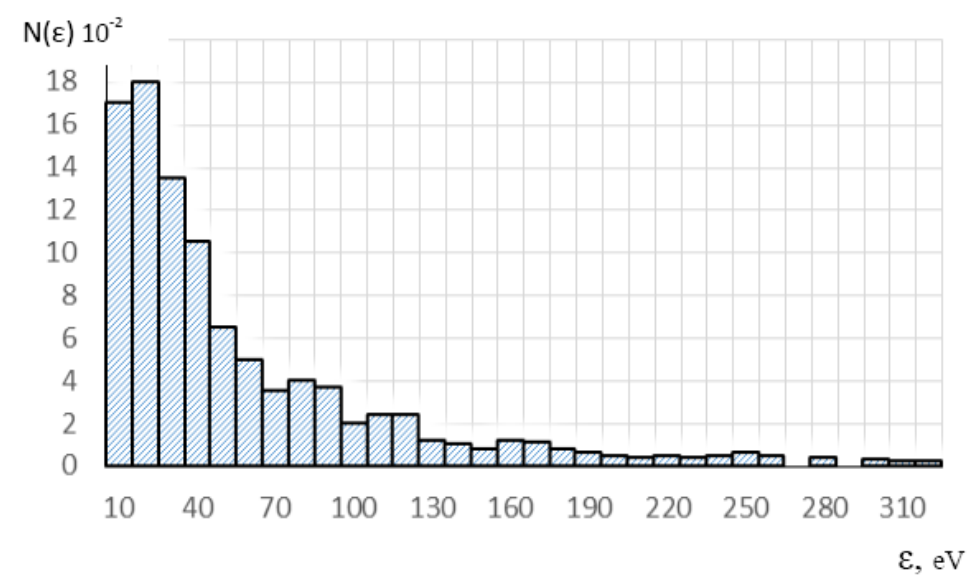

Fig. 4. Energy distribution.

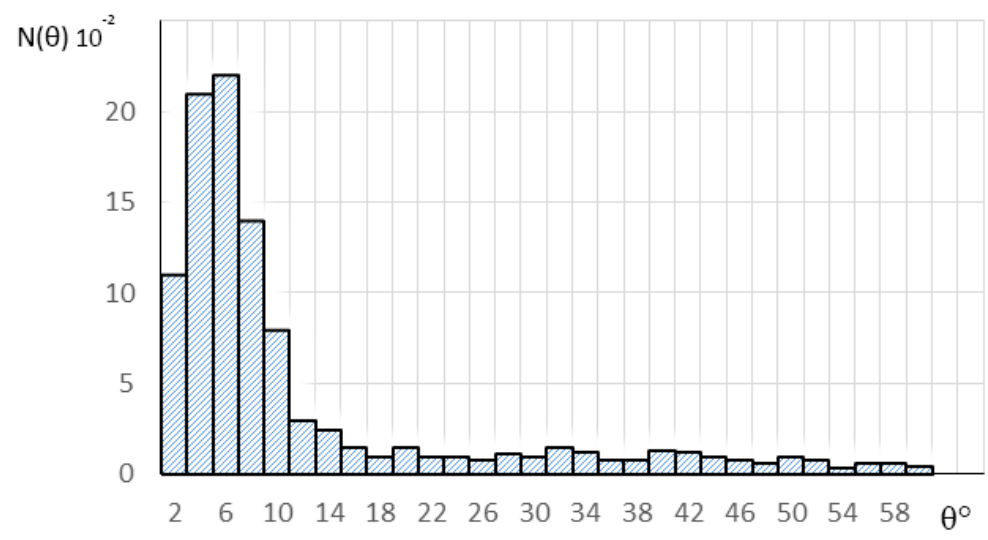

Fig. 5. Angular distribution.

The current density distribution in the image of the channel were determined from the arrival coordinates of the electron on the screen where the energy and angular distributions are used as the initial parameters in the further calculations. Fig. 6 and Fig. 7 demonstrate the results of calculations of the current density distribution for the uniform and nonuniform electrostatic fields at the exit of the channel. Corresponding MTFs are shown in Fig. 8 where curves 1 and 2 represent MTFs for the nonuniform field at the exit of the channel without (curve 1) and with (curve 2) the emission from the contact conducting layer. Curve 3 is obtained for the uniform electrostatic field at the exit of the channel. Fig. 8 demonstrates the effect of the field penetration into the channel and the electron emission from the contact conducting layer. It is obvious that the electrostatic lens at the exit of the channel has a significant influence on the electron trajectories and the spatial resolution. 
The lens, formed by the penetration of the MCP-screen gap field into a channel, depends on the depth $h$ of the conducting coating at the output of the channel. The described model was used to investigate the effect of the length $h$ on the current density distribution in the channel image on the screen. A set of MTFs was calculated for different $h$ values, and was replotted as a family of $N=f(h, T)$ curves (Fig. 9), where $N$ is the spatial frequency (in the relative unit) at which the contrast is equal to $T$. (Value of $h$ is normalized to the channel diameter $d$ ).

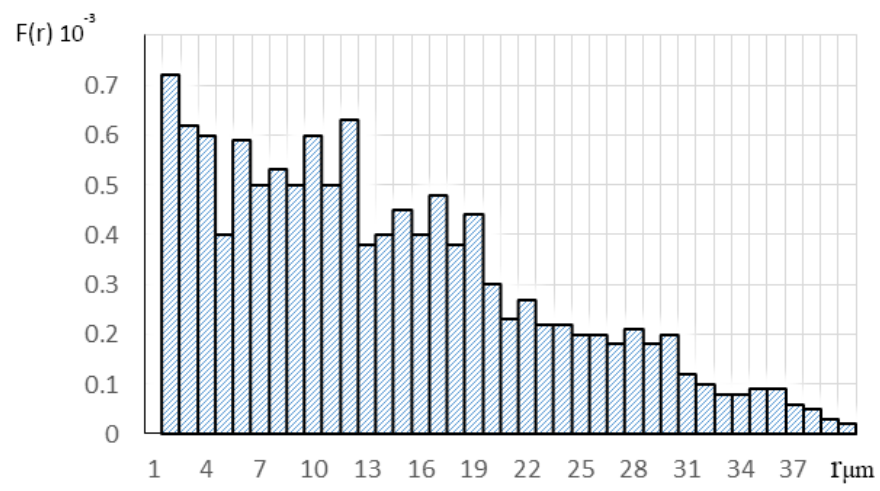

Fig. 6. Current - density distribution for the uniform field.

As seen from Fig. 9, the contrast $T$ at all spatial frequencies increases monotonically with an increase of the length $h$. This effect is explained by the fact that with an increase in the depth $h$ of the contact layer the electrostatic field of the MCP-screen gap penetrates deeper into the channel, focuses the electron beam, and increases the image contrast. Moreover, the exit aperture of the electron beam is decreased with an increase of $h$, i.e. the coating at the output of the channel acts as a limiting diaphragm.

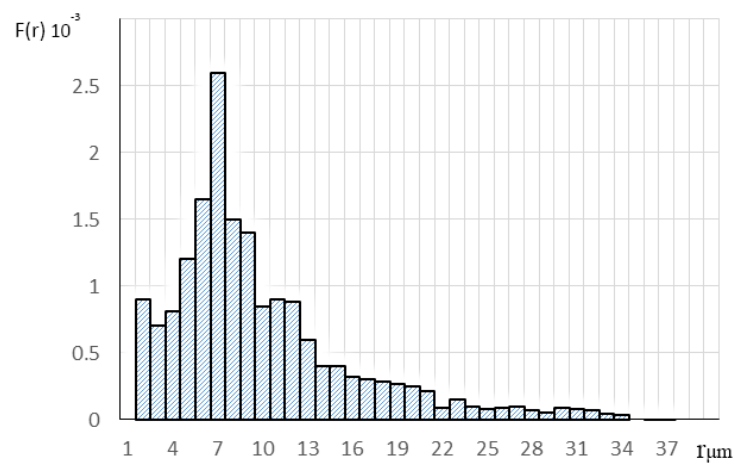

Fig. 7. Current - density distribution for the nonuniform field.

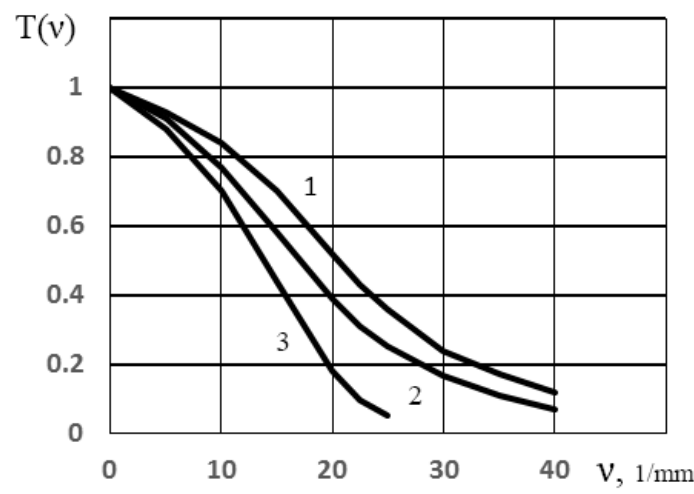

Fig. 8. MTFs of the MCP - screen system. 
However, the increase in the sputtering depth leads to a reduction of the gain due to the "settling" of electrons on the coating. Fig. 10 shows the dependence of the electron loss on the sputtering depth $h$. The electron loss was defined as the ratio of the difference in the number of electrons at the entrance of the sputtered region and the electrons at the output of the channel to the number of electrons at the entrance of the sputtered region (the emission from the coating was taken into account). Therefore, it is necessary to compromise between the MTF and the gain in choosing the length of the contact conducting layer.

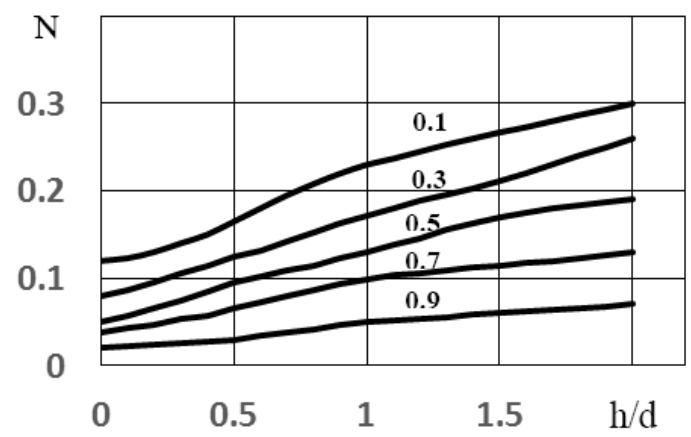

Fig. 9. Dependence of the spatial frequency $N$ on the length of the conducting layer $h$ for different contrasts.

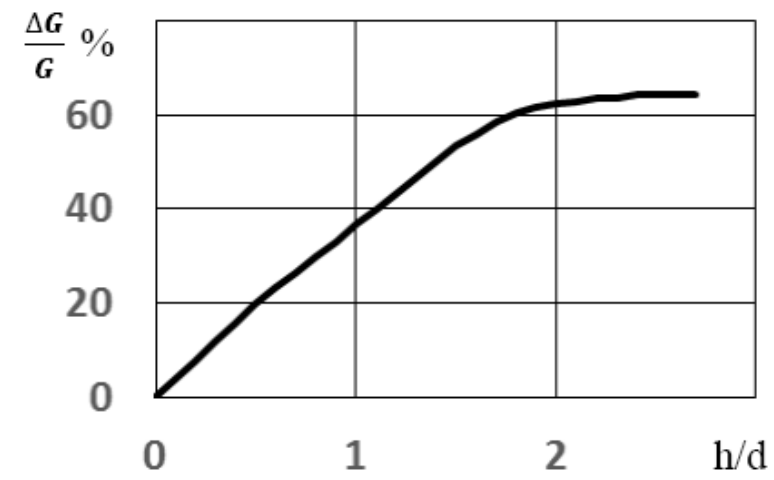

Fig. 10. Dependence of the electron loss on the sputtering depth $h$.

\subsection{Spatial Resolution of the Electron Optical System and the Device}

To provide the best image quality in the inverter imaging systems with MCP the image plane must be flat to match the micro channel plate, and the plate position should coincide with the surface of the best focus [3]. Fig. 11 represents the meridional trajectories of the electron beam near the screen calculated as described in section 2.1. It is seen that the image contrast at the point of the best focus $z_{f}$ will behigher than the image contrast at the cross-over position $z_{m}$. To evaluate the image contrast and precisely define the position of the surface of the best focus in the device, the MTF in the image plane for the point on the axis of the symmetry was calculated using the approach described in section 2.3.

The curvature of the image surface has been calculated as a total combination of minimal cross sections of the electron beams (cross-over positions), emitted from different points of the photocathode. Fig. 2 shows the central trajectories of the electron beams where coordinates of the minimal cross sections show the curvature of the image surface. It is seen that the image surface is not flat, and does not coincide with the channel plate. It leads to the nonuniform quality of the image on the screen.

To improve the image quality, the numerical experiments have been conducted for different radii of the spherical photocathode and distances from the photocathode to the anode. The numerical experiments show (Fig. 12) that the radius of the photocathode affects the curvature and the position of the image 
surface. It is seen that the relative radius of the photocathode, $\mathrm{R}=0.625$, provides the nearly flat image surface, and determines the position of the MCP in the device.

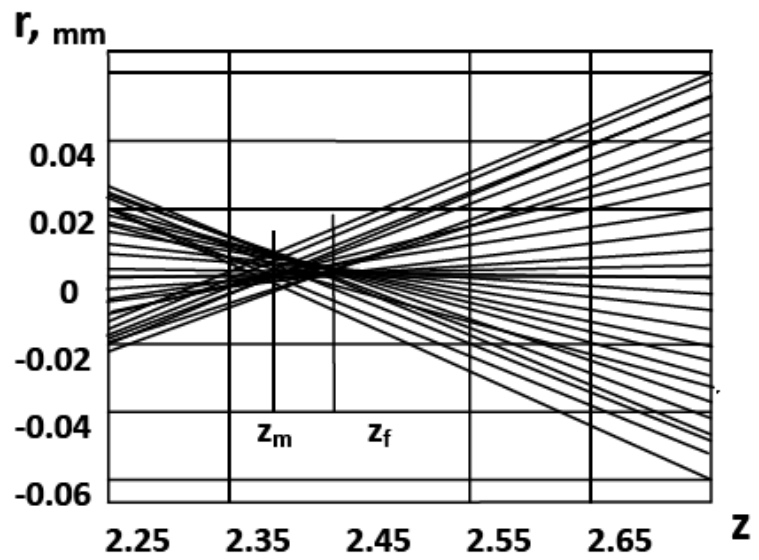

Fig. 11. Electron trajectories near the screen.

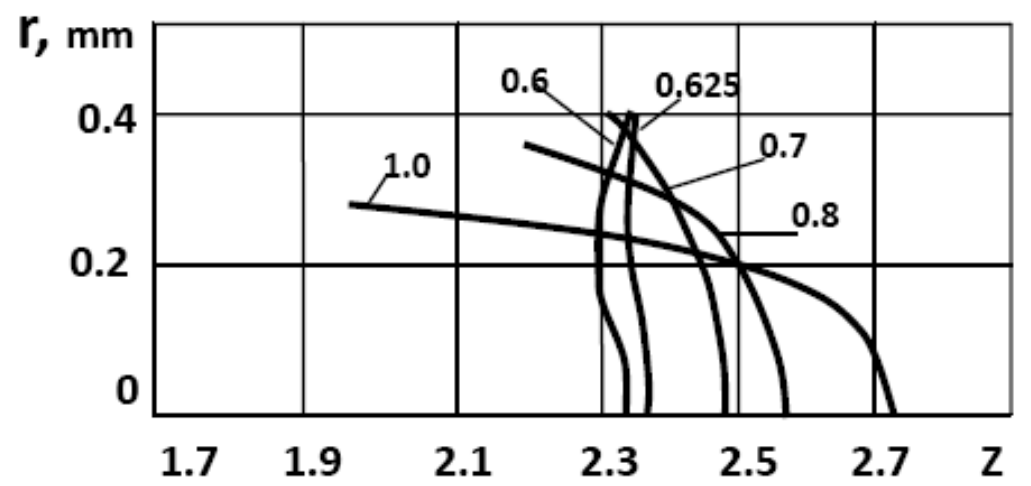

Fig. 12. The curvature of the image surfaces for different radii of the photocathode.

The final MTF of the image system is the product of the MTFs of the EOS and MCP. Fig. 13 shows the MTFs of the EOS (curve 1), MCP (curve 2) and the total MTF of the image device (curve 3), where the solid curves represent calculations $(d=10 \mu \mathrm{m})$ and dashed curve represents the experiment $(\mathrm{d}=12 \mu \mathrm{m}$ [12]) for the MCP-screen gap D=0.4 mm.

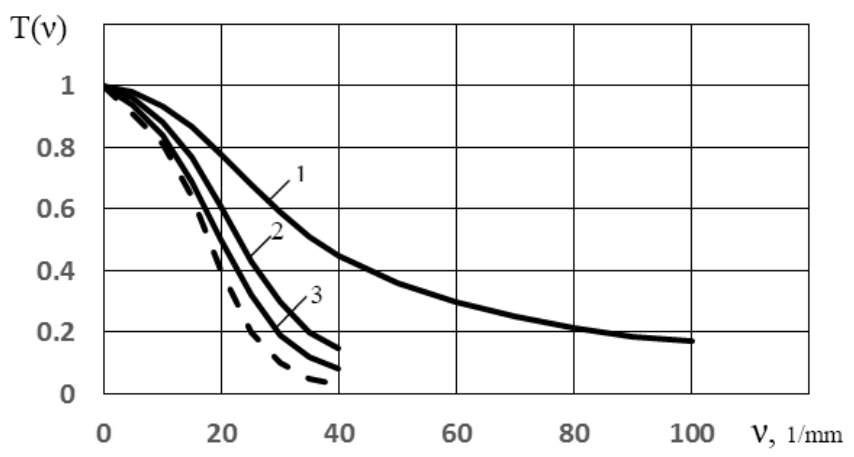

Fig. 13. MTFs of the EOS (curve 1), MCP-screen (curve 2) and the total MTF of the image device (curve 3).

\section{Conclusion}

The angular, energy and current-density distribution of the channel image on the screen have been 
calculated. The MTFs of the EOS, MCP-screen system and the total MTF of the device have been determined. It has been shown that the electrostatic field penetration into the channel at the exit significantly effects the image quality. The effect of the length of the contact conducting layer on the field penetration and MTF has been evaluated. It has also been shown how the emission from the conducting layer affects the resolution. The optimal parameters of EOS which provide a flat image surface to coincide with the channel plate have been defined. Position of the best focus in the electron beam, what provides the highest resolution, has been determined. Finally, the total MTF of the imaging device has been calculated.

\section{References}

[1] Clarke, J. A. (1973). Measuring the MTF of channel image intensifiers. Acta Electron, 16(1), 33-41.

[2] Schagen, P. (1975). Image converters and intensifiers. J. Phys. E.: Sci.Instr., 8, 153-160.

[3] Shymanska, A. V. (2011). Numerical analysis of electron optical system with microchannel plate. J. Comput. Electron, 10, 291-299.

[4] Wiza, J. L. (1979). Microchannel plate detectors. Nucl. Instr. Methods A, 162, 587-601.

[5] Shymanska, A. V., \& Evdokimov, V. N. (1985). Effect of parameters of multidyne screen system on image quality. Sov. J. Opt. Technol., 52(7), 393-394.

[6] Hoenderken, T. H., Hagen, C. W., Barth, J. E., Kruit, P., \& Nutzel, G. O. (2001). Influence of the microchannel plate and anode gap parameters on the spatial resolution of an image intensifier. J. Vac. Sci. Technol. B, 19(3), 843-850.

[7] Guest, A. J. (1971). A computer model of channel multiplier plate performance. Acta Electron, 14, 79-97.

[8] Shymanska, A. V. (2010). Computational modeling of stochastic processes in electron amplifiers. J. Comput. Electron, 9, 93-102.

[9] Yakobson, A. M. (1966). Estimation of the multiplication coefficient of a secondary electron multiplier with a continuous dynode. Radiotekh. Electron, 11, 1813-1825.

[10] Miroshnikov, M. M. (1983). Theoretical Foundations of Electron-optical Devices. Mechanical Engineering, Leningrad.

[11] Tyutikov, A. M., \& Tsoy, L. B. (1976). Optic. Mechan. Industry, 2, 20-23.

[12] Emberson, D. L., \& Holmshaw, R. T. (1973). The design and performance of an inverting channel image intensifier. Acta Electron, 16(1), 23-32.

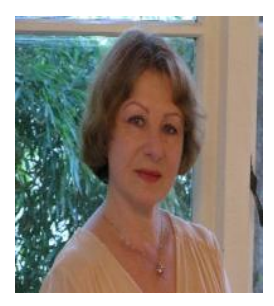

Alla Shymanska has her BEng in electronics and the $\mathrm{PhD}$ in mathematics and physics (St Petersburg). She has a long research and teaching experience in areas of physical electronics and charged particle optics at different research centers and universities in Russia and Ukraine. Currently she continues her research and teaches range of papers in mathematics and computer science at School of Computer and Mathematical Sciences of Auckland University of Technology, Auckland, New Zealand. 